\title{
Field-dependant hopping conduction in silicon nanocrystal films
}

\author{
M. A. Rafiq, ${ }^{1, a), b)}$ Z. A. K. Durrani, ${ }^{2, a)}$ H. Mizuta, ${ }^{3, a)}$ M. M. Hassan, ${ }^{4}$ and S. Oda ${ }^{1, a)}$ \\ ${ }^{1}$ Quantum Nanoelectronics Research Centre, Tokyo Institute of Technology, O-Okayama, Meguro-ku, Tokyo \\ 152-8552, Japan \\ ${ }^{2}$ Department of Electrical and Electronic Engineering, Imperial College London, South Kensington \\ Campus, London SW7 2AZ, United Kingdom \\ ${ }^{3}$ Nanoscale Systems Integration Group, School of Electronics and Computer Science, University of \\ Southampton, Southampton SO17 1BJ, United Kingdom \\ ${ }^{4}$ Pakistan Institute of Engineering and Applied Sciences, Islamabad 45650, Pakistan
}

(Received 17 July 2008; accepted 6 November 2008; published online 18 December 2008)

\begin{abstract}
We investigate the electric field dependence of hopping conduction in $300 \mathrm{~nm}$ thick films of $\sim 8 \mathrm{~nm}$ diameter silicon nanocrystals. The hopping conductivity $\sigma$ follows a $\ln (\sigma) \propto 1 / T^{1 / 2}$ dependence with temperature $T$, explained by a percolation hopping conduction model. At high fields $F>\sim 1$ $\times 10^{5} \mathrm{~V} / \mathrm{cm}$, the hopping conductivity follows a $\ln (\sigma) \propto F^{1 / 2}$ dependence. This dependence is investigated using the concept of "effective temperature," introduced originally by Shklovskii for hopping conduction in disordered materials. () 2008 American Institute of Physics.
\end{abstract}

[DOI: $10.1063 / 1.3050332]$

\section{INTRODUCTION}

In recent years, there has been great interest in systems of disordered material formed by semiconductor nanocrystals embedded in insulating matrices. ${ }^{1-6}$ Semiconductor nanocrystals, naturally formed by material synthesis, provide a means for the "bottom up" fabrication of nanoscale devices of increased functionality. In particular, silicon nanocrystals (SiNCs) are of great interest, as silicon is the material of choice for device fabrication. SiNCs have now been used to fabricate a variety of nanoscale devices, including singleelectron transistors, ${ }^{7}$ nonvolatile memories, ${ }^{8}$ electron emitters using SiNCs films $(60 \mathrm{~nm}-1.5 \mu \mathrm{m}),{ }^{9}$ and optoelectronic devices. ${ }^{1}$

Conduction in nanocrystal films at low temperatures occurs by a hopping transport mechanism, ${ }^{4,10}$ a process that can depend upon both temperature and applied electric field. This is due to the fact that in a random hopping system, carrier jumps to localized states at higher energies can be facilitated either by phonon interaction or by a reduction in the hopping energy barrier by an external electric field. There are, however, only few studies of the field dependence of hopping conduction in semiconductor nanocrystals. ${ }^{10}$ In particular, the field dependence of hopping conduction in films consisting of semiconductor nanocrystals dispersed in insulating matrices remains to be investigated in detail.

In our previous work, we investigated the conduction mechanism in thin films of SiNCs surrounded by $\mathrm{SiO}_{2}$ shells, prepared by plasma decomposition of silane. ${ }^{1}$ At electric field $F>\sim 1 \times 10^{5} \mathrm{~V} / \mathrm{cm}$ and temperatures $T$ from 40 to $200 \mathrm{~K}$, the conductivity $\sigma$ followed a $\ln (\sigma)$ versus $1 / T^{1 / 2}$ dependence, ${ }^{11}$ explained by the percolation hopping conduction model developed by Šimánek. ${ }^{12}$ At temperatures greater

\footnotetext{
a) Also at SORST JST (Japan Science and Technology).

${ }^{b}$ Also at National Centre for Nanotechnology, Pakistan Institute of Engineering and Applied Sciences, Islamabad, Pakistan. Electronic mail: aftab@cantab.net.
}

than $200 \mathrm{~K}$, space charge limited current has been observed. ${ }^{13}$ Recently, we reported the effect of SiNC diameter change on hopping conduction across SiNC films. ${ }^{14}$ However, the electric field dependence of hopping conduction across these films remained to be investigated.

In this paper, we investigate the electric field dependence of hopping conduction in SiNC films. We characterize our films using $\mathrm{Al} / p$-Si/SiNC film/Al diodes, where the SiNC film thickness is $\sim 300 \mathrm{~nm}$. Each SiNC in the film is $\sim 8 \mathrm{~nm}$ in diameter, and is encapsulated by a $\sim 1.5 \mathrm{~nm} \mathrm{SiO}{ }_{2}$ shell. From 200 to $40 \mathrm{~K}$, a $\ln (\sigma)$ versus $1 / T^{1 / 2}$ dependence is observed, similar to our previous work. At electric fields $F>$ $\sim 1 \times 10^{5} \mathrm{~V} / \mathrm{cm}$, the hopping conduction follows a $\ln (\sigma)$ versus $F^{1 / 2}$ dependence. This behavior is investigated using the concept of "effective temperature" developed by Shklovskii. ${ }^{15}$

\section{EXPERIMENT}

$\mathrm{The} \mathrm{Al} / p-\mathrm{Si} / \mathrm{SiNC}$ film/Al diodes were prepared by depositing SiNC films on $p$-Si substrates, similar to our previous devices. ${ }^{11,13}$ SiNC deposition was at room temperature, using the plasma decomposition of $\mathrm{SiH}_{4}{ }^{1}$ Figure 1(a) shows a scanning electron micrograph of the SiNC film. The SiNC film thickness is $\sim 300 \mathrm{~nm}$, and the SiNCs are $8 \pm 1 \mathrm{~nm}$ in diameter. ${ }^{1}$ Each SiNC is covered by a $\sim 1.5 \mathrm{~nm}$ thick $\mathrm{SiO}_{2}$ shell. Figure 1(b) shows a transmission electron micrograph of a $\sim 10 \mathrm{~nm}$ diameter SiNC. The $\mathrm{Al} / p-\mathrm{Si} / \mathrm{SiNC}$ film/Al diodes were fabricated using mesa structures in the SiNC film, defined by electron beam lithography and reactive ion etching, similar to our previous work. ${ }^{11,13}$ The diode area, determined by the top $\mathrm{Al}$ contact area, varied from 35 $\times 35$ to $200 \times 200 \mu \mathrm{m}^{2}$.

\section{RESULTS AND DISCUSSIONS}

Our electrical characteristics were measured using a cryogenic temperature probe station (BCT-43MDC, from Nagase \& Co. Ltd., Japan), and an Agilent 4156A parameter 

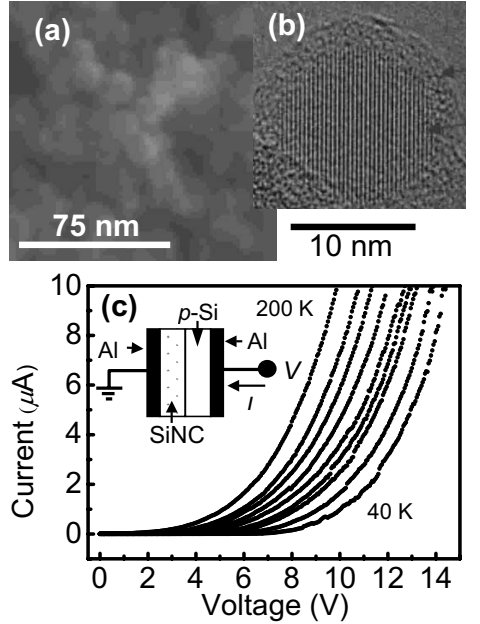

FIG. 1. (a) Scanning electron micrograph of the SiNC film. (b) TEM image of a $10 \mathrm{~nm}$ diameter SiNC. (c) $I V$ characteristics of device C from 40 to 200 $\mathrm{K}$ with a temperature step of $20 \mathrm{~K}$.

analyzer. A positive voltage is applied to the $p$-Si substrate [inset to Fig. 1(c)] and conduction occurs by hole-injection from the substrate. We measure $\sigma$ in four devices, devices A, $\mathrm{B}, \mathrm{C}$, and D, with areas of $35 \times 35,65 \times 65,100 \times 100$, and $175 \times 175 \mu \mathrm{m}^{2}$, respectively. Our characteristics were measured from 200 to $40 \mathrm{~K}$, with a temperature step of $20 \mathrm{~K}$. The current-voltage (IV) curves [Fig. 1(c)] were rectifying and asymmetric. We observe a $\ln (\sigma)$ versus $1 / T^{1 / 2}$ temperature dependence in all four devices, similar to the characteristics reported in our earlier work. ${ }^{11}$ Figure 2(a) shows the data for device $\mathrm{C}$, for three different values of electric field. Here, the electric field $F$ is defined as ratio of the applied voltage $V$ to our SiNC film thickness $(300 \mathrm{~nm})$.
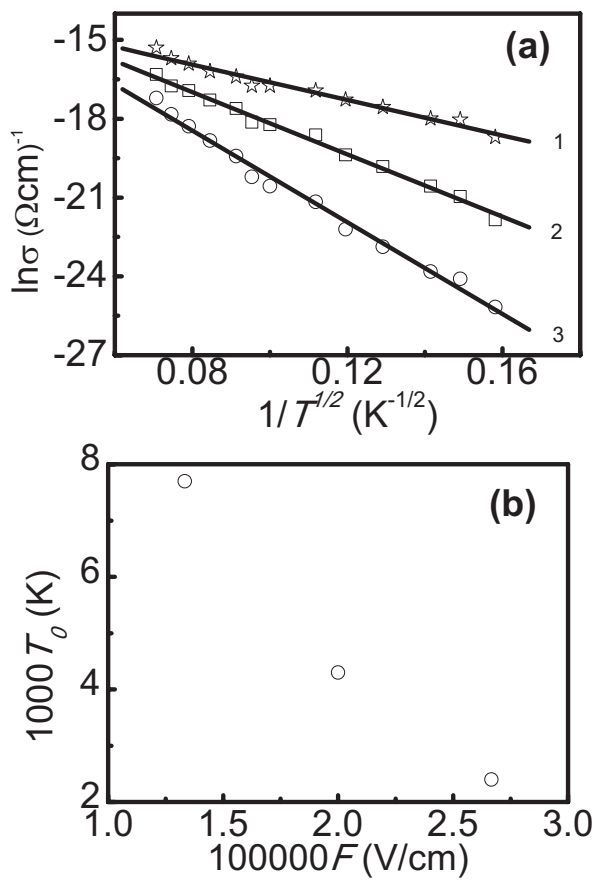

FIG. 2. (a) $\ln (\sigma)$ vs $1 / T^{1 / 2}$ plot for device $\mathrm{C}$ at fields (1) $F=1.3$ $\times 10^{5} \mathrm{~V} / \mathrm{cm},(2) 2 \times 10^{5} \mathrm{~V} / \mathrm{cm}$, and (3) $2.7 \times 10^{5} \mathrm{~V} / \mathrm{cm}$. (b) Variation in $T_{0}$ as a function of field $F$.
The temperature dependence may be explained by the Šimánek model of percolation hopping conduction, ${ }^{15}$ where thermally activated carrier tunneling between adjacent nanocrystals, i.e., nearest neighbor hopping, is considered. The nanocrystal size $d$ and separation $s$ are regarded as random, uncorrelated variables. The hopping activation energy $E_{a}$ is taken to be the energy difference between the first electron levels $E_{1}$ on neighboring nanocrystals. Here, $E_{1}=E_{c}+E_{d}$, where $E_{c}$ is the single-electron charging energy and $E_{d}$ is the quantum confinement energy. According to this mechanism, the conductivity is given by

$$
\sigma \propto \exp \left[-\left(\frac{T_{0}}{T}\right)^{1 / 2}\right] .
$$

At low bias, the characteristic temperature $T_{0}$ is a constant of the material and is given by

$$
T_{0}=\frac{2 P_{c} s_{\max } E_{a, \max }}{k_{B} a} .
$$

Here, $k_{B}$ is the Boltzmann constant, $P_{c}$ is the percolation threshold, $s_{\max }$ is the maximum particle separation, $a$ is the carrier wave function decay length in the insulating matrix, and $E_{a, \max }$ is the maximum activation energy for hopping. $P_{c}$ is unknown for SiNCs films. However according to Ziman, ${ }^{16}$ in both ordered and disordered lattices, $P_{c}$ follows the empirical formula $P_{c} \approx 1.5 / Z$, where $Z$ is the coordination number. Here we use an average value of $P_{c}=0.25$ (corresponding to a simple cubic lattice) as an approximation.

In Fig. 2(a) as $F$ increases, $T_{0}$ reduces. Figure 2(b) shows the reduction in $T_{0}$ as a function of electric field $F$. Therefore, increasing $F$ reduces the effect of temperature on $\sigma$, i.e., at higher $F$ there is a smaller change in $\sigma$ over the same range of temperature. Equation (2) suggests that $T_{0}$ would reduce if $E_{a, \max }$ reduces. This is possible as increasing $F$ reduces the hopping energy barriers. ${ }^{10}$ It is difficult to measure $T_{0}$ at fields below $F \sim 1 \times 10^{5} \mathrm{~V} / \mathrm{cm}$ due to reduction in current levels and an increase in the noise. The reduction in current levels is caused by the applied voltage dropping below the threshold voltage [Fig. 1(c)].

Equation (2) may be used to determine the theoretical value of $T_{0}=1 \times 10^{4} \mathrm{~K}$, described in detail in our previous work. ${ }^{11}$ This value is valid for low bias, where $E_{a, \max }$ is not perturbed by $F$. We may compare the theoretical value of $T_{0}$ to our experimental values at low bias, extracted from the slope of the $\ln (\sigma)$ versus $1 / T^{1 / 2}$ plot. At $F=1.33$ $\times 10^{5} \mathrm{~V} / \mathrm{cm}, T_{0}$ for devices A, B, C, and D is $1.24 \times 10^{4}$, $0.81 \times 10^{4}, 0.77 \times 10^{4}$, and $0.83 \times 10^{4} \mathrm{~K}$, respectively. The average value of $T_{0}$ is $0.91 \times 10^{4} \mathrm{~K}$. The areas of devices $\mathrm{A}$, $\mathrm{B}, \mathrm{C}$, and $\mathrm{D}$ is $35 \times 35,65 \times 65,100 \times 100$, and 175 $\times 175 \mu \mathrm{m}^{2}$, respectively The average value of $T_{0}$ varies $\pm 35 \%$ with respect to mean value of $T_{0}$. The observed variation in the value of $T_{0}$ may be associated with nonuniformity within the SiNC film. The average value of $T_{0}$ is in good agreement with our theoretical value of $1.0 \times 10^{4} \mathrm{~K}$.

We now investigate the effect of $F$ on $\sigma$. Figure 3(a) shows a $\ln (\sigma)$ versus $F^{1 / 2}$ plot for device $\mathrm{C}$ at fields $F>$ $\sim 1 \times 10^{5} \mathrm{~V} / \mathrm{cm}$, as the temperature varies from 40 to $160 \mathrm{~K}$ in $20 \mathrm{~K}$ steps. It is seen that the conductivity follows a $\ln (\sigma)$ 

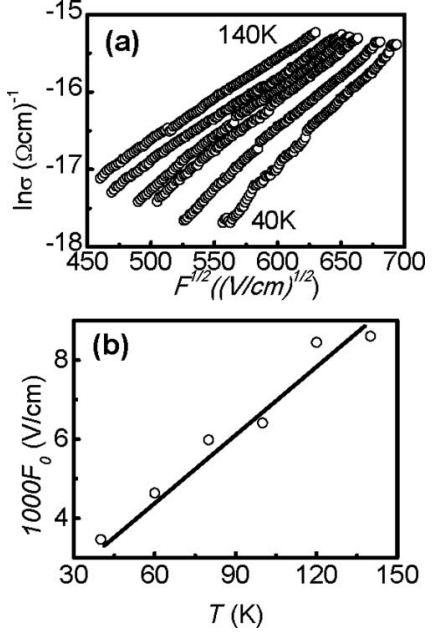

FIG. 3. (a) $\ln (\sigma)$ vs $F^{1 / 2}$ plot for device C. $T$ is varied from 40 to $140 \mathrm{~K}$, in $20 \mathrm{~K}$ steps. (b) $F_{0}$ vs $T$ plot, where $F_{0}$ is the slope of the $\ln (\sigma)$ vs $F^{1 / 2}$ characteristics given in (a). The solid line is aid to the eyes.

versus $F^{1 / 2}$ dependence. Furthermore, the slope of these characteristics reduces with increasing temperature, consistent with the behavior of Fig. 2(a). We observe similar characteristics in all four devices. Figures 2(a) and 3(a) illustrate that the conductivity in our films depends on both electric field and temperature. The electric field dependence of the conductivity in our devices can be expressed as

$$
\sigma \propto \exp \left[+\left(\frac{F}{F_{0}}\right)^{1 / 2}\right] .
$$

In our data, $F_{0}$ increases as the temperature increases from 40 to $\sim 140 \mathrm{~K}$.

Hohl et al. ${ }^{17}$ explained an $\ln (\sigma)$ versus $F^{1 / 2}$ dependence in films of cobalt nanoclusters surrounded by dielectric shells using the concept of effective temperature in hopping transport, introduced by Shklovskii. ${ }^{15,17}$ The morphology of our SiNC films is somewhat similar. The effective temperature model considers the influence of $T$ and $F$ on the hopping conductivity, using a single quantity $T_{\text {eff }}(T, F)$, the effective temperature. Following the approach of Hohl et al., $T_{\text {eff }}$ may be generally expressed as

$$
T_{\text {eff }}=\left[T^{\beta}+(A F)^{\beta}\right]^{1 / \beta} .
$$

Here the factor $A F$ has units of temperature and $\beta$ is a constant. In the expression given by Hohl et al., $A=\gamma e \alpha / k_{B}$, where $\alpha$ is the hopping length and $\gamma$ is a further constant. $A$ is therefore proportional to the hopping length. Equation (4) also uses the externally applied field $F$. Typically, $T_{\text {eff }}$ is defined using $F_{h}$, the field between two hopping sites, and $e \alpha F_{h}$ gives the energy gained by a hopping electron from $F_{h}{ }^{17,18}$ In Eq. (4), we assume that $F \propto F_{h}$, i.e., $F=c F_{h}$ and that the proportionality constant $c$ is included in $A$.

The dependence described by Eq. (3) can be obtained by substituting Eq. (4) for $T$ in Eq. (1), with $\beta=1 / 2$ and using $A F \ll T$. $^{17}$ This gives the following expression:

$$
\sigma \propto \exp \left[-\left(\frac{T_{0}}{T}\right)^{1 / 2}+\left(\frac{F}{F_{0}}\right)^{1 / 2}\right] .
$$

Here, $F_{0}=T^{2} / T_{0} A$. Equation (5) predicts $\ln (\sigma) \propto F^{1 / 2}$ at constant $T$, giving the field dependence of our data [Fig. $3(\mathrm{a})]$.

We investigate the effective temperature model further by investigating the temperature dependence of $F_{0}$. Figure 3(b) shows $F_{0}$ [extracted from the data of Fig. 3(a)] as a function of $T$, from 40 to $140 \mathrm{~K}$. Figure 3(b) shows that $F_{0}$ $\propto T$. However, in Eq. (5), $F_{0}=T^{2} / T_{0} A$ and if $A$ is constant, then $F_{0} \propto T^{2}$. Our data do not fit this dependence. However, if $A$ varies linearly with $T$, then this predicts our observed $F_{0}$ $\propto T$. In the effective temperature model, $A$ is proportional to the hopping length $\alpha$. We therefore suggest that in our SiNC films, $\alpha \propto T$. This is possible as increased temperature reduces the effect of the barrier heights between the SiNCs and therefore, a longer localization length becomes possible. ${ }^{10}$

We comment briefly on the possibility of the PooleFrenkel effect in our films. This also predicts a $\ln (\sigma)$ versus $F^{1 / 2}$ dependence, caused by the field-enhanced thermal excitation of trapped carriers. ${ }^{19}$ Our SiNC films are however strongly inhomogeneous, and in such a system, the PooleFrenkel effect may not be a suitable description. ${ }^{17}$ Furthermore, the Poole-Frenkel model leads to an unrealistic estimation of the dielectric constant of our film by two orders of magnitude.

\section{CONCLUSIONS}

In conclusion we investigated the electric field dependence of hopping conduction in SiNCs films, from 40 to 200 $\mathrm{K}$. We observe a $\ln (\sigma) \propto\left(F / F_{0}\right)^{1 / 2}$ dependence at fields $>1$ $\times 10^{5} \mathrm{~V} / \mathrm{cm}$, where $F_{0}$ varies linearly with temperature. This dependence is investigated using the effective temperature model for hopping transport in disordered materials.

${ }^{1}$ Y. Kanemitsu, S. Okamoto, M. Otobe, and S. Oda, Phys. Rev. B 55, R7375 (1997).

${ }^{2}$ T. A. Burr, A. A. Seraphin, E. Werwa, and K. D. Kolenbrander, Phys. Rev. B 56, 4818 (1997).

${ }^{3}$ L. Chen, W. Chen, and F. C. Hong, Appl. Phys. Lett. 86, 193506 (2005).

${ }^{4}$ M. Fujii, O. Mamezaki, S. Hayashi, and K. Yamamoto, J. Appl. Phys. 83, 1507 (1998).

${ }^{5}$ B. O. Dabbousi, M. G. Bawendi, O. Onitsuka, and M. F. Rubner, Appl. Phys. Lett. 66, 1316 (1995).

${ }^{6}$ H. E. Romero and M. Drndic, Phys. Rev. Lett. 95, 156801 (2005).

${ }^{7}$ A. Dutta, S. Oda, Y. Fu, and M. Willander, Jpn. J. Appl. Phys., Part 1 39, 4647 (2000)

${ }^{8}$ S. Tiwari, F. Rana, H. Hanafi, A. Hartstein, E. F. Crabbé, and K. Chan, Appl. Phys. Lett. 68, 1377 (1996).

${ }^{9}$ K. Nishiguchi, X. Zhao, and S. Oda, J. Appl. Phys. 92, 2748 (2002).

${ }^{10}$ D. Yu, C. Wang, B. L. Wehrenberg, and P. Guyot-Sionnest, Phys. Rev. Lett. 92, 216802 (2004).

${ }^{11}$ M. A. Rafiq, Y. Tsuchiya, H. Mizuta, S. Oda, S.Uno Z. A. K. Durrani, and W. I. Milne, J. Appl. Phys. 100, 014303 (2006).

${ }^{12}$ E. Šimánek, Solid State Commun. 40, 1021 (1981).

${ }^{13}$ M. A. Rafiq, Y. Tsuchiya, H. Mizuta, S. Oda, S. Uno, Z. A. K. Durrani, and W. I. Milne, Appl. Phys. Lett. 87, 182101 (2005).

${ }^{14}$ X. Zhou, K. Usami, M. A. Rafiq, H. Mizuta, Y. Tsuchiya, and S. Oda, J. Appl. Phys. 104, 024518 (2008).

${ }^{15}$ B. I. Shklovskii, Fiz. Tekh. Poluprovodn. (S.-Peterburg) 6, 2335 (1972).

${ }^{16}$ J. M. Ziman, J. Phys. C 1, 1532 (1968).

${ }^{17}$ G.-F. Hohl, S. D. Baranovskii, J. A. Becker, F. Hensel, S. A. Quaiser, and M. T. Reetz, J. Appl. Phys. 78, 7130 (1995).

${ }^{18}$ S. Marianer and B. I. Shklovskii, Phys. Rev. B 46, 13100 (1992).

${ }^{19}$ J. Frenkel, Phys. Rev. 54, 647 (1938). 